\title{
The Future of BPM: Flying with the Eagles or Scratching with the Chickens?
}

\author{
Peter Dadam \\ Institute of Databases and Information Systems, Ulm University, Germany \\ peter.dadam@uni-ulm.de
}

Service-oriented architectures, business process management (BPM) systems, and BPM in general receive a lot of attention these days and the number of articles which describe the benefits and great potential of these technologies has significantly increased. It is something like a second wave after the first (and short) workflow hype in the middle of the 90's. However, the contemporary hype in newspapers and IT magazines does not really reflect reality. In fact, much more companies are still thinking about whether and in which form they shall introduce these technologies rather than concretely performing projects in these fields. And many companies which have started respective projects are still in the phase of designing and implementing (web) services or in evaluating SOA platforms and repositories of different vendors; i.e., they are still not bringing (larger) processes into production. Nevertheless, expectations are very high: Everything will become easier and more flexible, implementation of crossorganizational processes will become business as usual, and process management systems will enable new kinds of process-aware applications which have to be performed manually today. In fact, BPM has a great potential. However, to realize this potential in practice, we have to face much more the challenges of the real world, we have to learn more seriously from how business processes are executed today, and we have to understand how actors deal with exceptional situations. It is not hard to predict what will happen with the current BPM hype if users discover that they cannot do much more with these technologies than with previous ones or, even worse, that they can do less. And no organization will accept to become inflexible. - It is partially up to us, whether BPM will become a big and sustainable success or whether it will share the fate of many other hypes (like Computer Integrated Manufacturing at the end of the 80's). This talk will present real-world examples from different domains to illustrate where we jump too short. It will use the ADEPT project [12] to show how stimulating it can be also from a research point of view to face the reality as it is.

\section{References}

1. Reichert, M., Dadam, P.: ADEPT flex - Supporting Dynamic Changes of Workflows Without Losing Control. J. of Intelligent Information Systems 10(2), 93-129 (1998)

2. Reichert, M., Rinderle, S., Kreher, U., Dadam, P.: Adaptive Process Management with ADEPT2. In: Proc. ICDE 2005, pp. 1113-1114 (2005) 